\title{
Vena cava defect repair using a polytetrafluoroethylene graft after a radical nephrectomy and vena cava resection: A case report
}

\author{
Volkan Izol, Mutlu Deger, Mustafa Zuhtu Tansug \\ Department of Urology, Faculty of Medicine, University of Çukurova, Adana, Turkey.
}

\begin{abstract}
Summary Introduction: The gold standard treatment for large renal masses is a radical nephrectomy and the removal of tumor thrombi from the large vessels. Here, we discussed the repair of a vena cava defect using a polytetrafluoroethylene (PTFE) graft after a radical nephrectomy and vena cava resection.

Case: A 69-year-old male patient presented to our clinic with right-sided pain and $10 \mathrm{~kg}$ of weight loss over the previous 3 months. The computed tomography showed that the right kidney was $23 \times 13 \mathrm{~cm}$ in size, with a $7 \times 6 \times 7 \mathrm{~cm}$ contrastenhanced mass at the renal ilum level. The patient underwent a radical nephrectomy, and the vena cava defect was repaired using a PTFE graft. There was also tumor infiltration in the proximal third of the left renal vein. The renal vein defect was also repaired using a PTFE graft, and the end of the graft was sutured to the vena cava graft at a right angle. The histopathological examination showed a Fuhrman grade 4 renal cell carcinoma (RCC) with focal sarcomatoid differentiation areas. Conclusions: The management of patients with RCCs and inferior vena cava (IVC) tumor thrombi should be planned with an experienced team, including a cardiovascular surgeon and liver transplantation team. In these patients, the comorbidities, life expectancy, and imaging methods should be considered for treatment planning in experienced centers. The tumor stage, probability of invasion, and patient's performance status should also be determined using magnetic resonance imaging during the preoperative period. Finally, the needs for a graft or tubular patch, sternotomy, and chemotherapeutic agents after the nephrectomy should be discussed using a multidisciplinary approach.
\end{abstract}

KEY WORDS: Radical nephrectomy; Vena cava; Renal cell carcinoma; Polytetrafluoroethylene; Graft.

Submitted 9 March 2019; Accepted 3 April 2019

\section{INTRODUCTION}

Renal cell carcinomas (RCCs) account for approximately $3 \%$ of all adult solid tumors and approximately $85 \%$ of all parenchymal kidney tumors. 1 After prostate and bladder tumors, RCCs are the third most commonly seen urological tumors, and they exhibit the highest mortality rate of all urological cancers. Although an RCC diagnosis can be made during the early stages with the widespread use of imaging modalities, $25 \%$ of the patients may have metastatic and venous involvement, extending from the renal vein to the right atrium. This is seen in 4-
$10 \%$ of the cases at the time of the diagnosis (2-4). Evaluating the tumor size, location, inferior vena cava (IVC) thrombus presence, adjacent organ involvement, lymph node involvement, and any distant metastases before performing an intervention is very important for determining the surgical margin and the patient's survival. Nephron sparing surgery or a radical nephrectomy, which is the standard treatment for an organ confined RCC, allows for a high-grade cure. Unfortunately, after a radical nephrectomy and tumor thrombus removal in the case of IVC tumors, the 5-year survival rate drops to 32$64 \%$ (5-8). However, the survival rate in patients who have undergone only radical nephrectomies, without removing any thrombi, declines significantly, and most of these patients die within the first year. Despite the significant improvements that have been made in targeted therapy in recent years, the most effective treatment for these patients is still surgery.

Although large renal masses can be removed safely with the developments that have been made in the preoperative diagnostic methods, anesthesia, and surgical techniques, especially after liver transplantation, morbidity and mortality rates ranging from $2.7-40 \%$ have been reported (9). There are three important stages involved in RCC and venous tumor thrombus surgery: renal artery ligation, avena cava tumor thrombectomy, and radical nephrectomy. Due to IVC tumor infiltration in certain rare cases, a resection of the infiltrating section of the IVC may also be necessary (10). After the resection and an adequate hepatic vena cava dissection, the vena cava can be anastomosed end to end, or the defect can be repaired with synthetic or homologous grafts.

Here, we have discussed a vena cava defect repair case in which a polytetrafluoroethylene (PTFE) graft was used after a radical nephrectomy and vena cava resection.

\section{Case report}

A 69-year-old male patient presented to our clinic with right-sided pain and $10 \mathrm{~kg}$ of weight loss over the previous 3 months. The ultrasonography showed that left kidney was normal, but a mass completely infiltrated the right kidney. The complete blood count and serum biochemistry values were normal, the Karnofsky Performance Scale Index was 80\%, and the patient's medical history did 
Figure 1.

MRI revealed a $10 \mathrm{~cm}$ mass compressing the liver at the right renal hilum level and extending to the midline paraaortic localization, filling the renal vein, vena cava with suspected wall invasion.

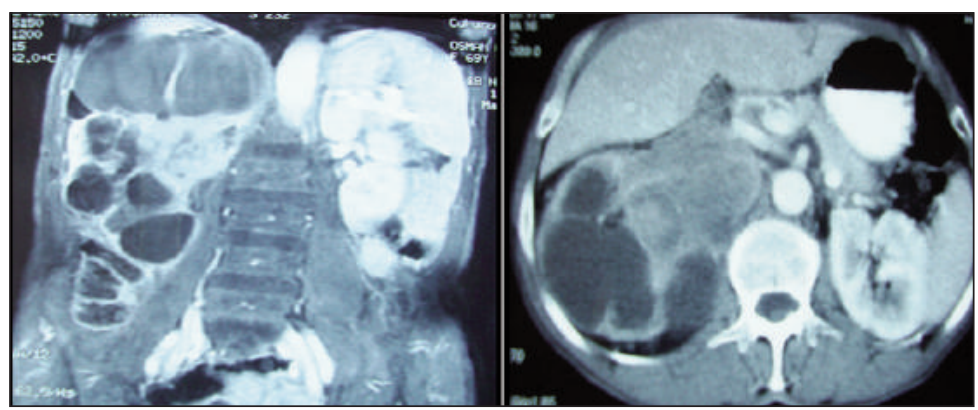

not include any significant features, with the exception of hypertension and smoking 60 packs/years.

Computed tomography (CT) scan showed that right kidney was $23 \times 13 \mathrm{~cm}$ in size, with a contrast-enhanced mass at the renal ilum level of $7 \times 6 \times 7 \mathrm{~cm}$ in size. Magnetic resonance (MR) imaging was used to determine the relationship between the mass surrounding the renal ilum and the vascular structures. The MR images revealed a $10 \mathrm{~cm}$ mass compressing the liver at the right renal ilum level, which extended to the para-aortic midline, filling the renal vein and vena cava, with suspected wall invasion (Figure 1). Bone scintigraphy and thorax tomography showed no metastases.

After patient evaluation, an experienced cardiovascular surgeon and liver transplantation team planned a radical nephrectomy, thrombectomy, and IVC resection and reconstruction. Following the necessary preparations,

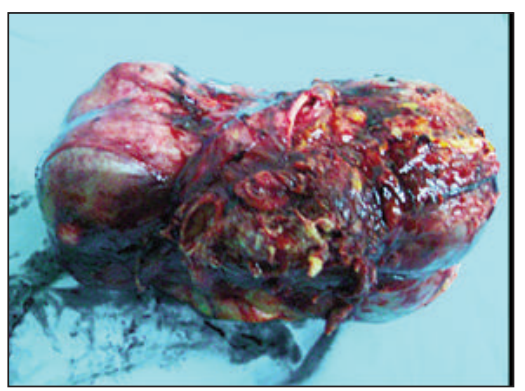

Figure 2.

The renal mass was removed to show the inferior part of the inferior vena cava.

\section{Figure 3.}

Approximately $13 \mathrm{~cm}$ vena cava defect was repaired using a $16 \mathrm{~mm}$ diameter $x 13 \mathrm{~cm}$ long PTFE graft achieving continuity of the inferior vena cava. The defect in the renal vein was repaired with a $10 \mathrm{~mm}$ diameter $x$ $4 \mathrm{~cm}$ long PTFE graft and the end of the graft was sutured to the vena cava graft at a right angle.

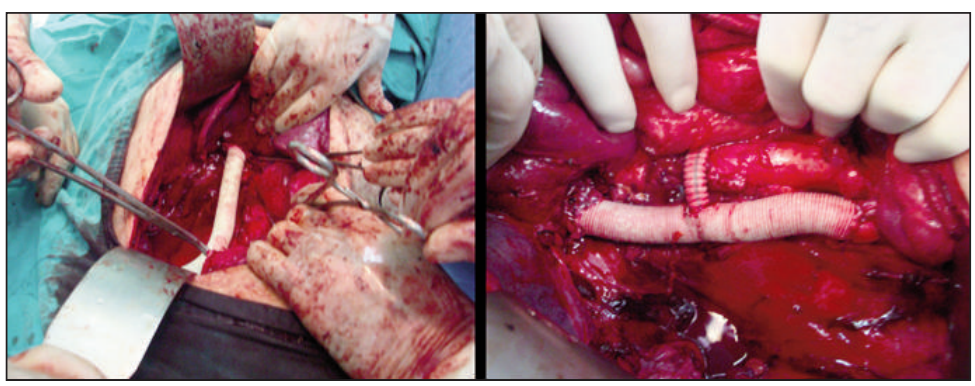

the surgery began with a transperitoneal subcostal incision in right semi-lumbar position under general anesthesia. When the colon was turned over and the retroperitoneal area was reached, a mass extending proximally to the bottom of the liver with the IVC could be seen. There was also tumor infiltration in the proximal third of the left renal vein. The hepatic vena cava was dissected, and vascular clamps were placed on the distal and proximal sections of the vena cava and on the renal vein of the opposite kidney. After controlling the renal arteries, the renal mass was removed to uncover the inferior section of the IVC (Figure 2). An approximately $13-\mathrm{cm}$ vena cava defect was repaired using a $16-\mathrm{mm}$ diameter, 13-cm long PTFE graft, achieving continuity of the IVC. The defect in the renal vein was repaired with a 10-mm diameter, 4-cm long PTFE graft, and the end of the graft was sutured to the vena cava graft at a right angle (Figure 3). The operation ended after the bleeding was controlled.

The operation time was 300 minutes, the estimated blood loss was $5,000 \mathrm{ml}$, and 14 erythrocyte transfusion units were given. The patient, who was extubated during the postoperative period, was admitted to the service without any problems, and low molecular weight heparin was administered. On the $3^{\text {rd }}$ postoperative day, this patient was started on oral intake; however, he developed acute right-sided pain on the 6th postoperative day, and a hematoma was detected in the operation zone. The hematoma was evacuated under general anesthesia, and no bleeding focus was found. Two blood transfusion units were administered, and the procedure was terminated. The preoperative creatinine level was $0.8 \mathrm{mg} / \mathrm{dl}$, the postoperative level increased to $2 \mathrm{mg} / \mathrm{dl}$, and upon discharge, it was $0.7 \mathrm{mg} / \mathrm{dl}$. The patient underwent drainage on the $9^{\text {th }}$ postoperative day, and he was discharged on the $14^{\text {th }}$ postoperative day.

The histopathological examination showed a Fuhrman grade 4 RCC with focal sarcomatoid differentiation areas. At the 1 month follow-up, the positron emission tomography $\mathrm{CT}$ of the operation region showed a large number of mass lesions consistent with metastases extending from the para-aortic region to the pelvic region. There was a recurrent mass in the posteromedial section of the liver, with suspicion of invasion. This patient was referred for a consultation in the medical oncology department, and interferon treatment was started. However, this patient died due to metabolic causes during the 6 th postoperative month.

\section{Discussion}

The gold standard treatment for large renal masses is a radical nephrectomy and tumor thrombus removal from the large vessels (if there are any). This process was first described by Berg in 1913, and since then, it has been applied as a standard treatment method $(11,12)$. When making surgical deci- 
sions, the metastasis-related symptoms (e.g., IVC syndrome, weight loss, hematuria, edema, and side pain), comorbidities (e.g., hypertension, heart failure, diabetes mellitus, chronic obstructive pulmonary disease, and secondary malignancy), patient's performance status, and patient's life expectancy must be taken into account. Thrombus removal with a radical nephrectomy may reduce the disease-related symptoms. This can lead to a better quality of life for the patient, even though it may not provide a curative treatment (12). Additionally, the administration of novel chemotherapeutic agents, such as tyrosine kinase inhibitors (e.g., sunitinib and sorafenib) and mammalian target of rapamycin kinase inhibitors (e.g., temsirolimus and everolimus or RAD001), after cytoreductive surgery can also extend the life expectancy of these patients (13). The most important issue when determining the prognosis involving a tumor thrombus is whether the tumor is invasive with regard to the vascular structures. If the tumor has invaded the vascular structures with a thrombus, the prognosis will be worse whether this patient has metastases or not. A good MR imaging examination and transesophageal echocardiography should be performed (during the operation, if necessary) to make this differentiation (14-16).

Surgery is not difficult for renal tumors with tumor thrombi in the subcortical IVC, and in majority of cases, minimal vena cava invasion can be treated using standard surgical approaches. If the thrombus does not occupy much space in the IVC, a radical nephrectomy alone can be performed, while milking the thrombus to the renal vein. However, more complicated surgical approaches are needed when the thrombus is at a higher level or the right atrium is extended. Although various surgical thrombus resection methods have been suggested, a cardiopulmonary bypass, deep hypothermia, and transient circulatory arrest are the ones most commonly used. In addition, various surgical maneuvers, such as hepatic mobilization, the Pringle maneuver, or a venovenous bypass, may be required. If vascular invasion is suspected and a surgical decision is made, a partial or total IVC resection and synthetic vascular grafting can be performed. However, these should only be performed in tertiary hospitals using a multidisciplinary approach, including an anesthetist with sufficient experience, a vascular surgeon, a liver transplant team, and a urologist (10-16)

When the tumor thrombus partially or totally infiltrates the IVC wall, the wall should be resected until a reliable surgical margin is reached; however, the indications for resection and the reconstructive methods to be used afterwards are not clearly defined. Some authors have argued against reconstruction after a suprarenal resection because collateral vessels, such as lumbar, epigastric, and vertebral arteries, may develop. However, other authors have suggested that major venous insufficiency may develop if it is not done. In general, if the IVC wall defect is less than $1 / 3$ of the wall diameter, a direct repair should be performed; if it is excessive, reconstruction should be performed with a PTFE or tubular graft.

The PTFE graft was first used by Sarti et al. in 1970, and it has been applied safely to patients with malignant tumors since that time (17).
The increased laparoscopic renal surgery experience in recent years has brought with it the feasibility of this method for patients with widespread IVC thromboses. In the past, a laparoscopic approach seemed to be relatively contraindicated in cases with a renal vascular tumor thrombosis. Nowadays, in some clinics, especially those experienced in laparoscopic surgery, tumor surgeries including level I thromboses and some cases level II can be performed laparoscopically. In some studies (despite their small number) it has been argued that a handassisted laparoscopic nephrectomy is safe if the thrombus is in the renal vein, and it can even be comparable to an open radical nephrectomy $(18,19)$. Depending on the experience of the clinic, the patient's risk factors, and the thrombus grade, an open or laparoscopic approach can be used in certain cases.

Despite the advances that have been made in preoperative imaging methods and the increase in surgical experience, the mortality rates during this operation range between $2.7 \%$ and $13 \%$. The most common causes of mortality are massive bleeding and pulmonary emboli $(12,20-22)$. In one study, 659 patients who underwent thrombectomies at the Mayo Clinic were evaluated retrospectively, and a 15\% complication rate was reported. The subgroup analysis revealed an increase in the complication probability as the thrombus level increased; as a result, the need for a multidisciplinary approach to reduce morbidity was emphasized (23).

\section{Conclusions}

The management of patients with RCCs that include IVC tumor thrombi should include an experienced team consisting of a cardiovascular surgeon and liver transplantation team in an experienced center. A tumor burden reduction with minimal morbidity to a maximum extent is very important for the patient's survival. Moreover, the tumor stage, invasion probability, patient's performance status, comorbidities, and life expectancy should be evaluated using imaging methods, such as MR imaging, during the preoperative period. The needs for a graft or tubular patch, sternotomy, and chemotherapeutic agents after the nephrectomy should all be discussed with a multidisciplinary team. The patient should also be informed about the operation and the planned procedures both before and after surgery.

\section{References}

1. Jacqmin D, van Poppel H, Kirkali Z, et al. Renal cancer. Eur Urol. 2001; 39:361-9.

2. Janzen NK, Kim HL, Figlin RA, et al. Surveillance after radical or partial nephrectomy for localized renal cell carcinoma and management of recurrent disease. Urol Clin North Am. 2003; 30:843-52

3. Pagano F, Dal Bianco M, Artibani W, et al. Renal cell carcinoma with extension into the inferior vena cava: problems in diagnosis, staging and treatment. Eur Urol. 1992; 22:200-203.

4. Marshall F, Dietrick D, Baumgartner W, et al. Surgical management of renal cell carcinoma with intracaval neoplastic extension above the hepatic veins. J Urol. 1988; 139:1166-72. 
5. Padovan RS, Perkov D, Smiljanic R, et al. Venous spread of renal cell carcinoma. Abdom Imaging. 2007; 32:530-7.

6. Schimmer C, Hillig F, Riedmiller $H$, et al. Surgical treatment of renal cell carcinoma with intravascular extension. Interact Cardiovasc Thorac Surg. 2004; 3:395-7.

7. Wotkowicz C, Wszolek MF, Libertino JA. Resection of renal tumors invading the vena cava. Urol Clin North Am. 2008; 35:657-71.

8. Kaplan S, Ekici S, Dogan R, et al. Surgical management of renal cell carcinoma with inferior vena cava tumor thrombus. Am J Surg. 2002; 183:292-9.

9. Hallscheidt P, Pomer S, Roeren T, et al. Preoperative staging of renal cell carcinoma with caval thrombus: is staging in MRI justified? Prospective histopathological correlated study. Urologe A. 2000; 39:36-40.

10. Duckett JW, Lifland JH, Peters PC. Resection of the inferior vena cava for adjacent malignant diseases. Surg Gynecol Obstet. 1973; 136:711-6.

11. Okada Y, Kumada K, Terachi T, et al. Long-term followup of patients with tumor thrombi from renal cell carcinoma and total replacement of the inferior vena cava using an expanded polytetrafluoroethylene tubular graft. J Urol. 1996; 155:444-7.

12. Rigaud J, Hetet JF, Braud G, et al. Surgical care, morbidity, mortality and follow-up after nephrectomy for renal cancer with extension of tumor thrombus into the inferior vena cava: retrospective study since 1990s. Eur Urol. 2006; 50:302-10.

13. Procopio G, Verzoni E, de Braud F. Targeted therapies and survival: what we can learn from studies in advanced renal cell carcinoma. Oncology. 2013; 84:39-42.

14. Sidana A, Goyal J, Aggarwal P, et al. Determinants of outcomes after resection of renal cell carcinoma with venous involvement. Int Urol Nephrol. 2012; 44:1671-9.

15. Boorjian SA, Sengupta S, Blute M. Renal cell carcinoma: vena caval involvement. BJU Int. 2007; 99:1239-44.

16. Kirkali Z, Van Poppel H. A critical analysis of surgery for kidney cancer with vena cava invasion. Eur Uro.l 2007; 52:658-662.

17. Sarti L. Total prosthetic transplantation of the inferior vena cava, with venous drainage restoration of the one remaining kidney on the graft, successfully performed on a child with Wilms' tumor. Surgery. 1970; 67:851-5.

18. Henderson A, Murphy D, Jaganathan K, et al. Rané Handassisted laparoscopic nephrectomy for renal cell cancer with renal vein tumor thrombus. Urology. 2008; 72:268-72.

19. Hoang AN, Vaporcyian AA, Matin SF. Laparoscopy-assisted radical nephrectomy with inferior vena caval thrombectomy for level II to III tumor thrombus: a single-institution experience and review of the literature. J Endourol. 2010; 24:1005-12.

20. Steahler $G$, Brkovic D. The role of radical surgery for renal cell carcinoma with extension into vena cava. J Urol. 2000; 163:1671-5.

21. Skinner DG, Pritchett TR, Lieskovsky G, et al. Vena caval involvement by renal cell carcinoma: surgical resection provides meaningful long-term survival. Ann Surg. 1989; 210:387-94.

22. Nesbitt JC, Soltero ER, Dinney CPN, et al. Surgical management of renal cell carcinoma with inferior vena cava tumor thrombus. Ann Thorac Surg. 1997; 63:1592-600.

23. Karnes RJ, Blute ML. Surgery insight: management of renal cell carcinoma with associated inferior vena cava thrombus. Nat Clin Pract Urol. 2008; 5:329-39

\section{Correspondence}

Volkan Izol, MD

Mustafa Zuhtu Tansug, MD

Department of Urology, Faculty of Medicine, University of Cukurova

Adana (Turkey)

Mutlu Deger, MD, FEBU (Correspondent Author)

drmutludeger@gmail.com

Department of Urology, Faculty of Medicine,

University of Çukurova, Adana 01330 (Turkey) 